\title{
An engineer's perspective on diversity
}

\section{Andre Sayles}

Andre H. Sayles, "An engineer's perspective on diversity," Proc. SPIE 9663, Eighth International Topical Meeting on Education and Training in Optics and Photonics, 96630S (6 October 2003); doi: 10.1117/12.2208447

SPIE Event: Eighth International Topical Meeting on Education and Training in Optics and Photonics, 2003, Tucson, Arizona, United States 


\title{
An engineer's perspective on diversity
}

\author{
Andre H. Sayles \\ Department of Electrical Engineering \& Computer Science, US Military Academy, West Point, NY 10996 \\ telephone: 845-938-2201; fax: 845-938-5956; e-mail: Sayles@usma.edu
}

\begin{abstract}
Organizational diversity has arrived at an important crossroads in our history. Over the past year, a somewhat novel approach to diversity based on organizational effectiveness has enabled many people from various backgrounds and levels of experience to better understand how diversity works and be able to assess both collective and individual progress.

(C)2003 Optical Society of America

OCIS codes: $(000.0000)$ General
\end{abstract}

\section{Introduction}

Over the past 50 years legislative actions and mandates have enhanced integration and created opportunities for many who may have otherwise been considered at a disadvantage. Today, we continue to seek out creative ways to meet diversity goals and, in some cases, compete for the attention of a small population within particular groups capable of bringing valuable differences to our organizations and institutions. We often do not view diversity as a process. Instead, experts tend to focus only on particular aspects of diversity. Sociologist offer powerful insights into the impact of equality and opportunity on various organized groups in society. Linguists and psychologists have thoroughly explained communication differences across ethnic and gender groups and the impact of those differences on work, school, and social environments. Historians describe how different groups have been treated over the years and how their contributions have shaped our nation. Various segments of our population sponsor observances and other productions that promote both recognition and understanding of a particular culture. On the contrary, engineers often prefer to work with processes, particularly those that allow for assumptions, inputs, and outputs. Unfortunately, not many engineers end up focusing on diversity issues as perhaps a secondary career field. This paper offers an engineering perspective on diversity by describing it as a process. The discussion includes a description of the success story dating back to the June 2002 creation of the concept.

\section{Discussion}

Diversity is a valuable organizational effectiveness resource. However, from a leadership or management perspective, the benefits of diversity do not come easy or happen naturally. The power of diversity in an organizational setting can be realized through a process that gives purpose to most of the things that we already do. We describe the diversity process as a pyramid with four parts defined as accepting differences, understanding differences, valuing differences, and role models. These terms are often used in diversity discussions and literature; however, our success is derived from the manner in which these phases or components have been defined and linked together to form a process. Since our process is well defined, we have also been able to take the somewhat unusual step of starting with a concise definition of diversity. With that definition in mind, we step through the process, describing each phase and showing how familiar diversity activities can be linked together to pursue goals, assess outcomes, and plan for the future in an organized manner. We define diversity as "differences in people that can have an impact on the effectiveness of an organization." Based on that definition, we argue that the first step in the process is accepting the notion that differences matter in a given organization - as opposed to everyone being viewed the same way regardless of the situation. The belief that differences matter should lead to a desire to understand those differences and how they can influence organizational effectiveness. Upon reaching a certain level of understanding, one can then transition to the higher category of valuing diversity as an organizational enhancement tool. Role models then bridge the gap between the present and the future.

\section{Conclusion}

This novel approach to organizational diversity has been presented in seminar format to a wide variety of audiences, including students, college staff and faculty members, equal opportunity professionals, professionals having equal opportunity as a secondary responsibility, human resource managers, and organizational leaders. Regardless of the venue, the outcome has been relatively uniform. Most individuals, including those who work as professionals in the field, say that the process brings about a new understanding of diversity. This diversity process is currently serving as a basis for re-engineering diversity programs in several military organizations. 